\title{
About the estimation of degree of coherence for circularly polarized waves
}

\author{
${ }^{1}$ Zenkova C.Yu., ${ }^{2}$ Gorsky M.P. and ${ }^{2}$ Gorodynska N.V. \\ ${ }^{1}$ Optics and Spectroscopy Department, Chernivtsi National University, \\ 2 Kotsyubinsky St., 58012 Chernivtsi, Ukraine, zenkova@itf.cv.ua \\ ${ }^{2}$ Department of Correlation Optics, Chernivtsi National University, \\ 2 Kotsyubinsky St., 58012 Chernivtsi, Ukraine
}

Received: 05.03.2010

After revision: 15.05.2010

\begin{abstract}
In this work we propose a technique of field polarisation modulation for determining degree of coherence for the circularly polarised waves. The processes of three-wave interaction are studied in connection with the problems of applied polarisation holography and, in particular, polarisation-sensitive diffraction gratings. We also demonstrate an important part of the reference circularly polarised wave in transformation of spatial polarisation distribution into a depth of visibility modulation of the resulting distribution, which can be metrologically estimated and analysed.
\end{abstract}

Keywords: polarisation, degree of coherence, state of polarisation, polarisation grating

PACS: $42.25 . \mathrm{Kb}, 42.25 . \mathrm{Hz}$

UDC: 535.41

\section{Introduction}

Spatial periodical polarisation modulation of resulting field distributions represents one of important manifestations of coherence of the superposing fields and diagnostic indicators of their coherence [1-3]. The depth and the level of this modulation are related to degree of coherence and correlation of the superposing fields. Interconnections between the degree (level) of polarisation modulation of the resulting field and the coherence characteristics have been thoroughly studied within the framework of Stokes polarimetric approach in the approximation of plane waves $[1,2]$.

Manifestations of coherence of the superposing wave for the case when the longitudinal $z$-component of the field has to be taken into consideration and the polarisation modulation of the resulting field occurs in the plane of incidence have been studied in a number of works [3,4]. The possibilities for measuring the field coherence function and the metrological principles of correlation in the optical irradiation field, through estimation of the degree (level) of the field polarisation modulation, have been justified in the framework of this approach $[5,6]$. As a consequence, the above works suggest a technique for determining the degree of coherence of linearly polarised fields, when the polarisation is distributed in a single plane, the plane of observation. The 
present work extends the methods suggested and demonstrates that they may be also used for the circularly polarised fields when the two polarised distributions are formed in two mutually orthogonal planes.

In particular, involving of a third (reading) wave allows one to reproduce information about the polarisation distribution in the recording plane and derive a coherence degree of the two orthogonally polarised waves, thus transforming polarisation distribution into the intensity one. The polarisation type of the third reading wave should be chosen according to a spatial arrangement of polarisation distribution under test. When two mutually orthogonal linearly polarised waves are superposed, the reading wave has to be plane and linearly polarised, because the polarisation distribution occurs in a single plane, the plane coinciding with the incidence one. On the other hand, one should choose a circularly polarised reading wave in case when the circularly polarised waves are interacting. Being represented by two linearly polarised waves, this reading wave provides information about the two spatial polarisation distributions, which are formed in two different planes, the incidence one and the plane perpendicular to it.

Thus, in the present study we suggest to extend the resources of metrological use of the spatial polarisation modulation field technique in estimating the coherence of superposing waves, by means of consideration of circularly polarised interacting waves, in addition to superposition of waves linearly polarised in the incidence plane. Those results would allow applying the theory of coherence to metrological uses and, in particular, would surely be useful in the applied polarisation holography and investigations of polarisation-sensitive systems, e.g. biological objects. In order to produce a wave with a desired wave front configuration and to produce a given polarisation, up to a vortex, one can employ a technique proposed in [7].

It is known that vectorial (or polarisation) holographic gratings are produced by using a polarisation-modulated interference pattern over a conventional intensitymodulated one. The polarisation modulation is achieved via interference between asymmetrically polarised beams. Most of the works in this field have so far been directed at vectorial gratings obtained with the interference of orthogonal left and right circularly polarised beams [8-14]. An interesting and appealing property of these devices is their polarisation-sensitive diffraction efficiency. In fact, this polarisation selectivity depends exclusively on the ellipticity of incident probe-beam polarisation [8]. At the same time, linear photoinduced anisotropy would as well be sufficient for the fabrication of these devices. When using linearly polarised light, an optical anisotropy may be generated in polymer films containing photoactive groups, anisotropically photorefractive polymer systems, liquid crystals, etc. A photo-control of molecular orientation of such materials is of great interest for the optical data storage and production of, e.g., birefringent films and highly functionalised optical devices. Although any necessary spatial patterns of molecular reorientation may, in principle, be designed for the highly functionalised optical devices, novel types of those devices should use controllability of reorientation direction of mesogenic molecules. 
Here a polarisation holographic technique represents one of sophisticated methods for solving the problem [15-17]. In frame of this technique, two coherent light waves with the orthogonal polarisations are used as writing beams. The interference pattern for the waves of equal amplitudes and orthogonal (linear or circular) polarisations is characterised with a constant intensity and a polarisation state, which is periodically modulated in space. As a rule, interaction of two orthogonal linearly polarised waves or two orthogonal circularly polarised waves of the opposite handedness is studied. Besides, polarisation gratings based on the orthogonal circularly polarised beams have been widely preferred over those using orthogonal S- and P-polarised beams, even though the latter configuration is the only one allowing formation of purely vectorial holographic gratings $[18,19]$. While the interaction of mutually orthogonal linearly polarised waves is appointed to producing the purely polarisation gratings, the interaction of orthogonally propagating circularly polarised waves of the same handedness produces not only $2 \mathrm{D}$ polarised gratings, but also 3D amplitude-phase gratings, thus enabling one to control simultaneously a whole set of diffracted radiation parameters.

Utilization of an appropriate recording medium allows one to produce polarisation gratings for controlling both the polarisation state and the propagation direction of the interacting waves. Due to availability of a large variety of proper photosensitive recording materials $[9,11,13]$, the orthogonal circularly polarised waves are widely used when making the polarisation gratings. Photochrome films on the basis of bacteriorhodopsin (biochrome) represent an example of such a medium. These films may be used for recording the interference gratings written by orthogonally polarised beams, i.e. for implementation of the polarisation holography. Then the necessary light intensity is two orders of magnitude smaller than that required for recording the polarisation gratings in dyes.

On the other hand, the information contained in the polarisation distribution of interacting circular waves of the same polarisations can enrich essentially the ideas about the properties of optical fields. In the present work we report on the results of computer simulations for the superposition of circularly polarised waves. Our results provide novel facilities for determining the degree of coherence of the writing interacting waves and extend the recourses for forming spatial polarisation and amplitude-phase diffraction elements using the orthogonal circularly polarised waves.

\section{Results of computer simulation}

Let us consider a general enough case of the interference of circularly polarised waves, with an angle $2 \theta$ between the initial waves $\left(\vec{E}^{(1)}\right.$ and $\left.\vec{E}^{(2)}\right)$ and the third reference wave $\left(\vec{E}^{(3)}\right)$ propagating perpendicular to the recording plane (see Fig. 1).

The modulated electric field is formed as a result of superposition of $x$ - and $z$ components $\left(\vec{R}_{1}\right)$ and $y$-and $z$-components $\left(\vec{R}_{2}\right)$. The electric field distribution for the $x$ and $z$-components in the observation plane is given by 


$$
\begin{aligned}
& \vec{R}_{1}=\left[\begin{array}{c}
E_{x}^{(1)} \exp \left[-i \sigma_{1}\right] \cos \theta+E_{x}^{(2)} \exp \left[-i \sigma_{1}\right] \cos \theta+E_{x}^{(3)} \exp \left[-i \sigma_{1}\right] \\
E_{z}^{(1)} \exp \left[i\left(\varphi_{1}^{\prime}-\sigma_{1}\right)\right]+E_{z}^{(2)} \exp \left[i\left(\varphi_{2}^{\prime}-\sigma_{1}\right)\right]+E_{z}^{(3)} \exp \left[i\left(\varphi_{3}^{\prime}-\sigma_{1}\right)\right]
\end{array}\right] \times \\
& \exp [i(\beta z-\omega t)] \text {, }
\end{aligned}
$$

where $\sigma_{1}=k n x \sin \theta, \quad k=\left|\vec{k}_{1}+\vec{k}_{2}+\vec{k}_{3}\right|$ and $\beta=k n \cos \theta$. Here the phase differences between the components of the interfering waves in the recording plane are $\varphi_{1}^{\prime}=\varphi_{x 1}-\varphi_{z 1}, \varphi_{2}^{\prime}=\varphi_{x 2}-\varphi_{z 2}$ and $\varphi_{3}^{\prime}=\varphi_{x 3}-\varphi_{z 3}, E_{x}, E_{y}, E_{z}$ denote the polarisation components of the incident waves and $\varphi_{x i}, \varphi_{y i}, \varphi_{z i}$ the phases of the corresponding components. In a quite similar manner, for the $y$ - and $z$-components one can obtain the following expression:

$$
\vec{R}_{2}=\left[\begin{array}{c}
E_{y}^{(1)} \exp \left[-i \sigma_{2}\right] \cos \theta+E_{y}^{(2)} \exp \left[-i \sigma_{2}\right] \cos \theta+E_{y}^{(3)} \exp \left[-i \sigma_{2}\right] \\
E_{z}^{(1)} \exp \left[i\left(\varphi_{1}^{\prime \prime}-\sigma_{2}\right)\right]+E_{z}^{(2)} \exp \left[i\left(\varphi_{2}^{\prime \prime}-\sigma_{2}\right)\right]+E_{z}^{(3)} \exp \left[i\left(\varphi_{3}^{\prime \prime}-\sigma_{2}\right)\right]
\end{array}\right] \times
$$

where $\sigma_{2}=k n y \sin \theta, \varphi_{1}^{\prime \prime}=\varphi_{y 1}-\varphi_{z 1}, \varphi_{2}^{\prime \prime}=\varphi_{y 2}-\varphi_{z 2}$ and $\varphi_{3}^{\prime \prime}=\varphi_{y 3}-\varphi_{z 3}$. The change, from zero to $\pi$, in the phase difference of the interfering waves at different points of the observation area brings about the corresponding change in the polarisation state and the spatial modulation of the polarisation distribution.

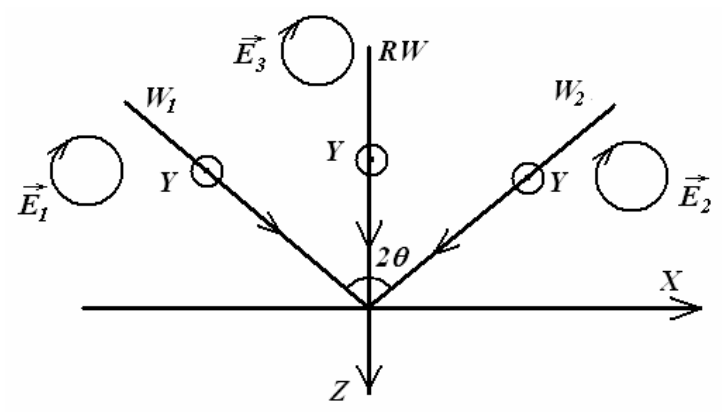

Fig. 1. Scheme of interaction of three waves. In general $W_{1}, W_{2}$ and $R W$ represent circularly polarised waves.

It is known $[5,6]$ that the time-averaged intensity distribution of a random electromagnetic field formed at some point of time $t$ by the sources $\vec{Q}_{1}, \vec{Q}_{2}, \vec{Q}_{3}$ and observed at a point $\vec{r}$ in the observation plane may be written as

$$
\begin{aligned}
I(\vec{r})= & \sum_{i j}\left\{\varphi_{i i}^{(1)}(\vec{r})+\varphi_{i i}^{(2)}(\vec{r})+\varphi_{i i}^{(3)}(\vec{r})+\right. \\
& 2 \sqrt{\operatorname{tr}\left[W\left(\vec{Q}_{1}, \vec{Q}, 0\right)\right] \operatorname{tr}\left[W\left(\vec{Q}_{2}, \vec{Q}_{2}, 0\right)\right]} \eta_{i j}^{(1,2)} \cos \left[\delta_{1}\right]+ \\
& 2 \sqrt{\operatorname{tr}\left[W\left(\vec{Q}_{1}, \vec{Q}_{1}, 0\right)\right] \operatorname{tr}\left[W\left(\vec{Q}_{3}, \vec{Q}_{3}, 0\right)\right]} \eta_{i j}^{(1,3)} \cos \left[\delta_{2}\right]+ \\
& \left.2 \sqrt{\operatorname{tr}\left[W\left(\vec{Q}_{2}, \vec{Q}_{2}, 0\right)\right] \operatorname{tr}\left[W\left(\vec{Q}_{3}, \vec{Q}_{3}, 0\right)\right]} \eta_{i j}^{(2,3)} \cos \left[\delta_{3}\right]\right\} .
\end{aligned}
$$


Here $i, j=x, y, z$, the parameters $\varphi_{i i}^{(m)}(\vec{r})=<E_{i}^{(m)}(\vec{r}, t) E_{i}^{*(m)}(\vec{r}, t)>($ with $m=1,2,3)$ describe the time-averaged intensities of the corresponding sources, the angle brackets denote the time averaging and the superscript $*$ stands for complex conjugation.

The coherence properties of the vector optical fields are described using the mutual coherency matrix $W\left(\vec{Q}_{m}, \vec{Q}_{n}, t\right)$ [20] characterising correlation of the fields at two different spatio-temporal points $\vec{Q}_{m}$ and $\vec{Q}_{n}$. This matrix is determined by the relation

$$
\begin{aligned}
& W\left(\vec{Q}_{m}, \vec{Q}_{n}, t\right)= {\left[\begin{array}{lll}
W_{x x}\left(\vec{Q}_{m}, \vec{Q}_{n}, t\right) & W_{x y}\left(\vec{Q}_{m}, \vec{Q}_{n}, t\right) & W_{x z}\left(\vec{Q}_{m}, \vec{Q}_{n}, t\right) \\
W_{y x}\left(\vec{Q}_{m}, \vec{Q}_{n}, t\right) & W_{y y}\left(\vec{Q}_{m}, \vec{Q}_{n}, t\right) & W_{y z}\left(\vec{Q}_{m}, \vec{Q}_{n}, t\right) \\
W_{z x}\left(\vec{Q}_{m}, \vec{Q}_{n}, t\right) & W_{z y}\left(\vec{Q}_{m}, \vec{Q}_{n}, t\right) & W_{z z}\left(\vec{Q}_{m}, \vec{Q}_{n}, t\right)
\end{array}\right]=} \\
& {\left[\begin{array}{rrr}
<E_{x}\left(\vec{Q}_{m}, t\right) E_{x}^{*}\left(\vec{Q}_{n}, t\right)> & <E_{x}\left(\vec{Q}_{m}, t\right) E_{y}^{*}\left(\vec{Q}_{n}, t\right)> & <E_{x}\left(\vec{Q}_{m}, t\right) E_{z}^{*}\left(\vec{Q}_{n}, t\right)> \\
<E_{y}\left(\vec{Q}_{m}, t\right) E_{x}^{*}\left(\vec{Q}_{n}, t\right)> & <E_{y}\left(\vec{Q}_{m}, t\right) E_{y}^{*}\left(\vec{Q}_{n}, t\right)> & <E_{y}\left(\vec{Q}_{m}, t\right) E_{z}^{*}\left(\vec{Q}_{n}, t\right)> \\
<E_{z}\left(\vec{Q}_{m}, t\right) E_{x}^{*}\left(\vec{Q}_{n}, t\right)> & <E_{z}\left(\vec{Q}_{m}, t\right) E_{y}^{*}\left(\vec{Q}_{n}, t\right)> & <E_{z}\left(\vec{Q}_{m}, t\right) E_{z}^{*}\left(\vec{Q}_{n}, t\right)>
\end{array}\right]=} \\
&<E_{i}\left(\vec{Q}_{m}, t\right) E_{j}^{*}\left(\vec{Q}_{n}, t\right)>,
\end{aligned}
$$

where the fields are determined by the random complex vectors $\vec{E}\left(\vec{Q}_{m}, t\right)$ and $\vec{E}\left(\vec{Q}_{n}, t\right)$ (often written in a simpler form, $\vec{E}^{(m)}$ or $\vec{E}^{(n)}$ ). Within the framework of this approach the parameter $\eta_{i j}^{(m, n)}=\frac{W_{i j}\left(\vec{Q}_{m}, \vec{Q}_{n}, t\right)}{\sqrt{\operatorname{tr}\left[W\left(\vec{Q}_{m}, \vec{Q}_{n}, 0\right)\right] \operatorname{tr}\left[W\left(\vec{Q}_{n}, \vec{Q}_{n}, 0\right)\right]}}$ defines the correlation degree of the field components, $\delta_{1}=k\left(R_{1}-R_{2}\right), \delta_{2}=k\left(R_{1}-R_{3}\right), \delta_{3}=k\left(R_{2}-R_{3}\right)$ are the phase differences of the corresponding fields at the recording plane, and $R_{1}=\left|\vec{r}-\vec{Q}_{1}\right|$, $R_{2}=\left|\vec{r}-\vec{Q}_{2}\right|, R_{3}=\left|\vec{r}-\vec{Q}_{3}\right|$ represent the distances between the point $\vec{r}$ and the source centres.

At first, let us consider a superposition of two absolutely coherent circularly polarised waves with the same rotation directions of their electric (magnetic) field vectors, which converge at the angle of $90^{\circ}$ (Fig. 2). This manifests itself in some spatial intensity distribution formed by the $x$ -,$y$ - and $z$-field components (for instance, $y$ component in our case). At the same time, the other two components, being mutually orthogonal, set some constant intensity (the background level) influencing the visibility of the pattern.

Then the visibility may be calculated in the same manner as for a standard case when two circularly polarised waves of the same handedness interfere at the angle of $90^{\circ}$ [5]:

$$
V=\frac{\left.\sqrt{\operatorname{tr}\left[W\left(\vec{Q}_{1}, \vec{Q}_{1}, 0\right)\right] \operatorname{tr}\left[W\left(\vec{Q}_{2}, \vec{Q}_{2}, 0\right)\right.}\right]}{\Phi_{y y}^{(1)}(r)+\Phi_{y y}^{(2)}(r)}\left|\eta_{y y}^{(1,2)}\right|,
$$

whereas the spatial polarisation distribution is given by Eq. (1), with $E_{x 3}=0$ and $E_{z 3}=0$. Because of the absence of any visibility distribution modulation (see Fig. 3), one 
cannot evaluate the degree of coherence of the initial waves in this case. The polarisation evolves from the linearly polarised state, through elliptical, and again into the linear state, thus repeating the distribution of polarisation modulation observed under the superposition of mutually orthogonal linearly polarised waves $[3-6,18,19]$.

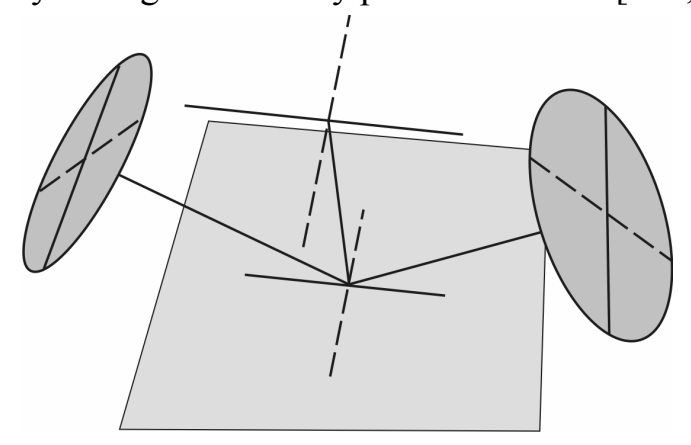

Fig. 2. Model interaction scheme for the two absolutely coherent circularly polarised waves of the same handedness and intensity.

Introduction of the reference wave propagating perpendicular to the observation plane, changes the spatial distributions of both the intensity and the polarisation state. The pattern of visibility distribution changes whenever the azimuth of the reference wave does (e.g., for the cases of $\alpha=0^{\circ}, 45^{\circ}$ and $90^{\circ}$ ).

Changes in the phase of the reference wave inside the interval $0.2 \pi$ result in periodical changes of the visibility of the recorded interference pattern, which follow the harmonic law $[5,6]$ :

$$
\begin{aligned}
V= & 2 \sum_{i j} \frac{\sqrt{\operatorname{tr}\left[W\left(\vec{Q}_{1}, \vec{Q}_{1}, 0\right] \operatorname{tr}\left[W\left(\vec{Q}_{2}, \vec{Q}_{2}, 0\right)\right]\right.}}{\varphi_{i i}^{(1)}(\vec{r})+\varphi_{i i}^{(2)}(\vec{r})+\varphi_{i j}^{(3)}(\vec{r})}\left|\eta_{i j}^{(1,2)}\right|+ \\
& 2 \sum_{i j} \frac{\sqrt{\operatorname{tr}\left[W\left(\vec{Q}_{1}, \vec{Q}_{1}, 0\right] \operatorname{tr}\left[W\left(\vec{Q}_{3}, \vec{Q}_{3}, 0\right)\right]\right.}}{\varphi_{i i}^{(1)}(\vec{r})+\varphi_{i j}^{(2)}(\vec{r})+\varphi_{i i}^{(3)}(\vec{r})}\left|\eta_{i j}^{(1,3)}\right| \cos \varphi_{1}+ \\
& 2 \sum_{i j} \frac{\sqrt{\operatorname{tr}\left[W\left(\vec{Q}_{2}, \vec{Q}_{2}, 0\right] \operatorname{tr}\left[W\left(\vec{Q}_{3}, \vec{Q}_{3}, 0\right)\right]\right.}}{\varphi_{i i}^{(1)}(\vec{r})+\varphi_{i i}^{(2)}(\vec{r})+\varphi_{i i}^{(3)}(\vec{r})}\left|\eta_{i j}^{(2,3)}\right| \cos \left[\varphi_{2}\right] .
\end{aligned}
$$

Here $\varphi_{1}$ and $\varphi_{2}$ are the phase differences between the two initial superposing waves and the reference wave. The visibility modulation depth (VMD) is determined as

$$
M=\max [V]-\min [V]=4 \sum_{m} \sum_{i j} \frac{\sqrt{\operatorname{tr}\left[W\left(\vec{Q}_{m}, \vec{Q}_{m}, 0\right] \operatorname{tr}\left[W\left(\vec{Q}_{3}, \vec{Q}_{3}, 0\right)\right]\right.}}{\varphi_{i j}^{(m)}(\vec{r})+\varphi_{i j}^{(3)}(\vec{r})}\left|\eta_{i j}^{(m, 3)}\right|(m=1,2) .
$$

Assuming that the reference wave is completely correlated with one of the initial waves (say, $\left|\eta^{(1,3)}\right|=1$ ), one can see that the VMD of the interference pattern characterises, up to a constant dependent on the intensity values, the degree of mutual coherence of the reference wave and the second of the initial waves, i.e. $M=\left|\eta^{(2,3)}\right|$. Taking into account the relation $\left|\eta^{(1,3)}\right|=1$, one concludes that $\left|\eta^{(2,3)}\right|=\left|\eta^{(1,2)}\right|$. Hence, by 
properly choosing the intensities of the interfering waves one can set the parameter $\left|\eta^{(1,2)}\right|$ such that it would be determined by the VMD of the interference pattern, $M=\left|\eta^{(1,2)}\right|$.

In other words, the change in the phase of the reference wave leads to a spatial intensity modulation. The influence of one of the components ( $x$-component) on the resulting intensity distribution increases. This allows to set the VMD and estimate the degree of coherence of the corresponding fields. Using a trial-and-error method for determining the amplitudes of the field components, one can obtain the maximum
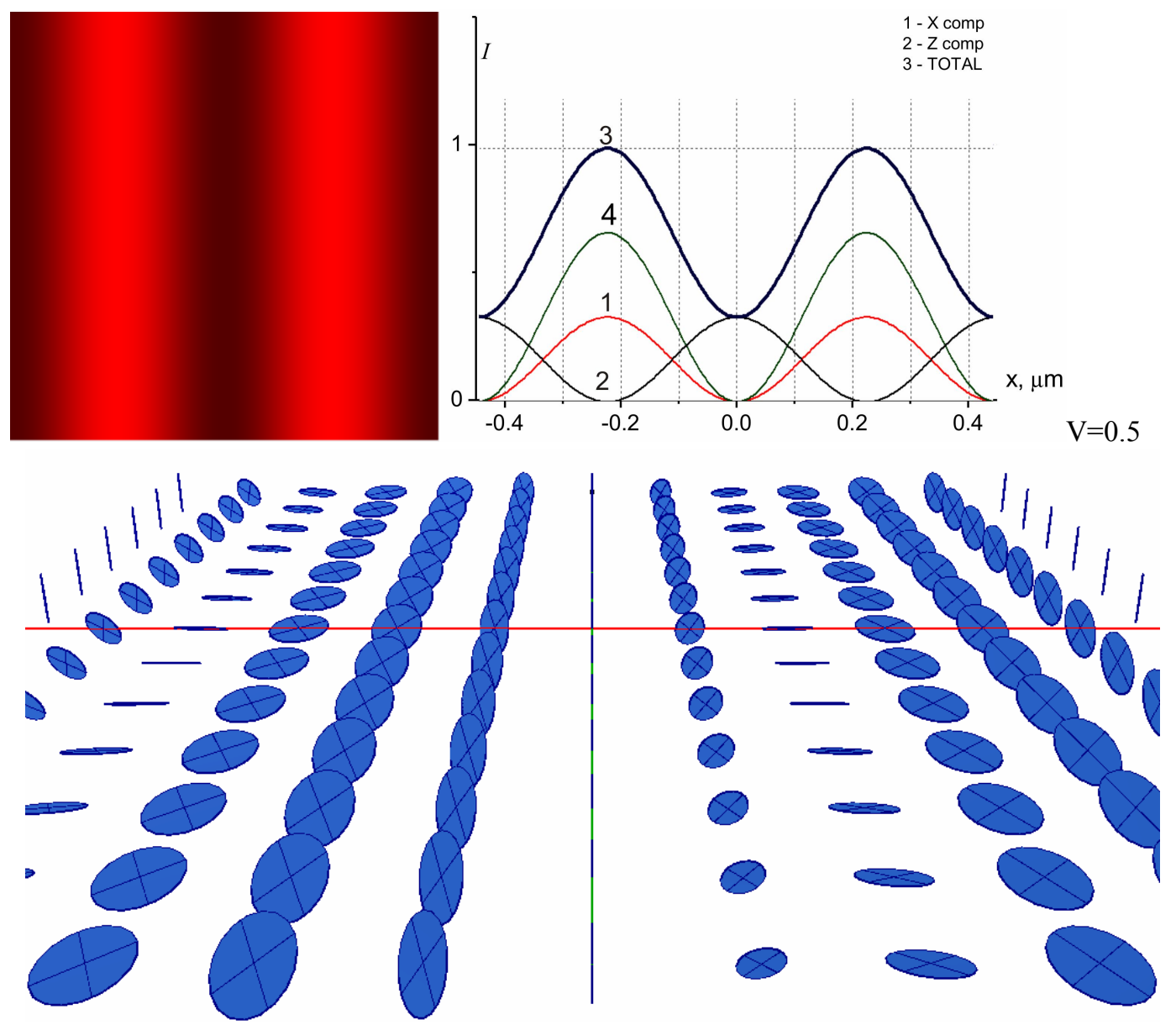

Fig. 3. Scheme that demonstrates intensity modulation, represented as dependences of the field intensities on the coordinate in the observation plane (upper right part), and spatial polarisation modulation (lower part) in the case of interaction of two coherent, orthogonally propagating and circularly polarised waves of the same handedness and intensity. Upper left part visualises interference of the waves. (1), (2) and (4) curves in the upper right part show respectively the modulations of the $z-, \quad x-$ and $y$-components, and (3) one corresponds to their superposition. Negative coordinates $x$ are due to the fact that the reference point "0" corresponds to the initial convergence point of the waves, where the resulting polarisation is linear and the phase difference zero. 
(minimum) VMD values at certain points of the observation area. The latter would enable estimating the coherence degree of the initial superposing waves with the formula $M=\left|\eta^{(1,2)}\right|$. Here the field polarisation state becomes more complex. One can distinguish the two distributions in the two mutually perpendicular planes, the incidence plane and the plane perpendicular to it, which are linked to the changes in the phase difference between the $x, z$ and $y, z$-field components at different points of the observation area.
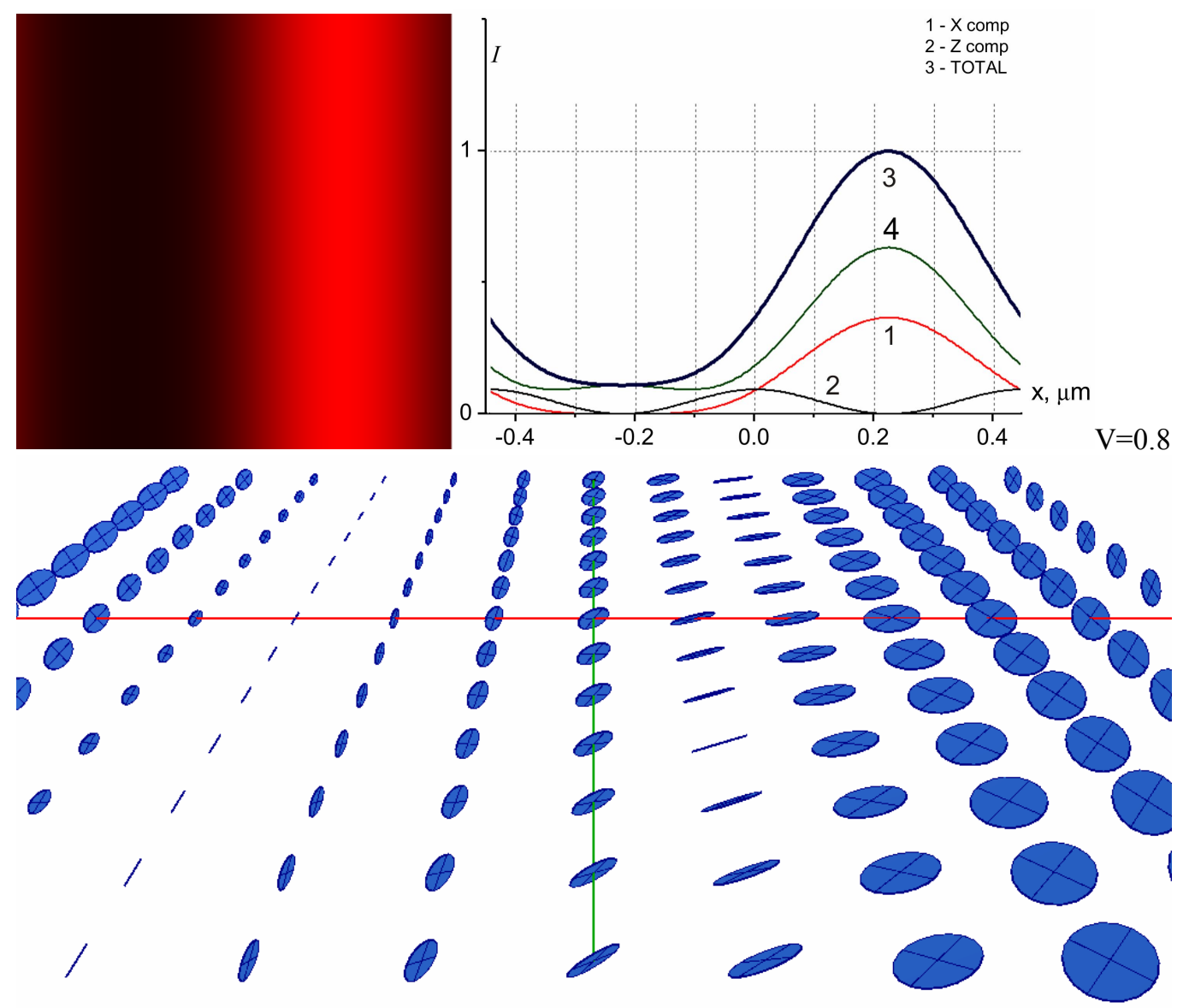

Fig. 4. Scheme demonstrating the intensity modulation and the spatial polarisation modulation for the case when two coherent, orthogonally propagating circularly polarised waves of the same handedness and intensity are superimposed with a third reference wave elliptically polarised at the azimuth of $45^{\circ}$. The notation is the same as in Fig. 3.

Introduction of a linearly polarised reference wave $\left(\alpha=0^{\circ}\right)$ changes the $x$ - and $y$ components of the resulting field and causes some intensity modulation, though with no means for obtaining simultaneously a zero value for all the components. An increase in the portion of the $x$-component leads to increasing visibility of the interference pattern due to decreasing background intensity, partially as a result of reduced influence of the $y$-component. In order to obtain the maximum VMD, it would be necessary to achieve 
simultaneously a maximum (minimum) value of all the three field components at some chosen points of the observation area, which might be expected for different polarisation of the reference wave and the corresponding choice of the intensities.

If the reference wave is elliptically polarised at the azimuth of $45^{\circ}$, we obtain the intensity distribution for which the VMD virtually corresponds to the coherence degree (see Fig. 4).
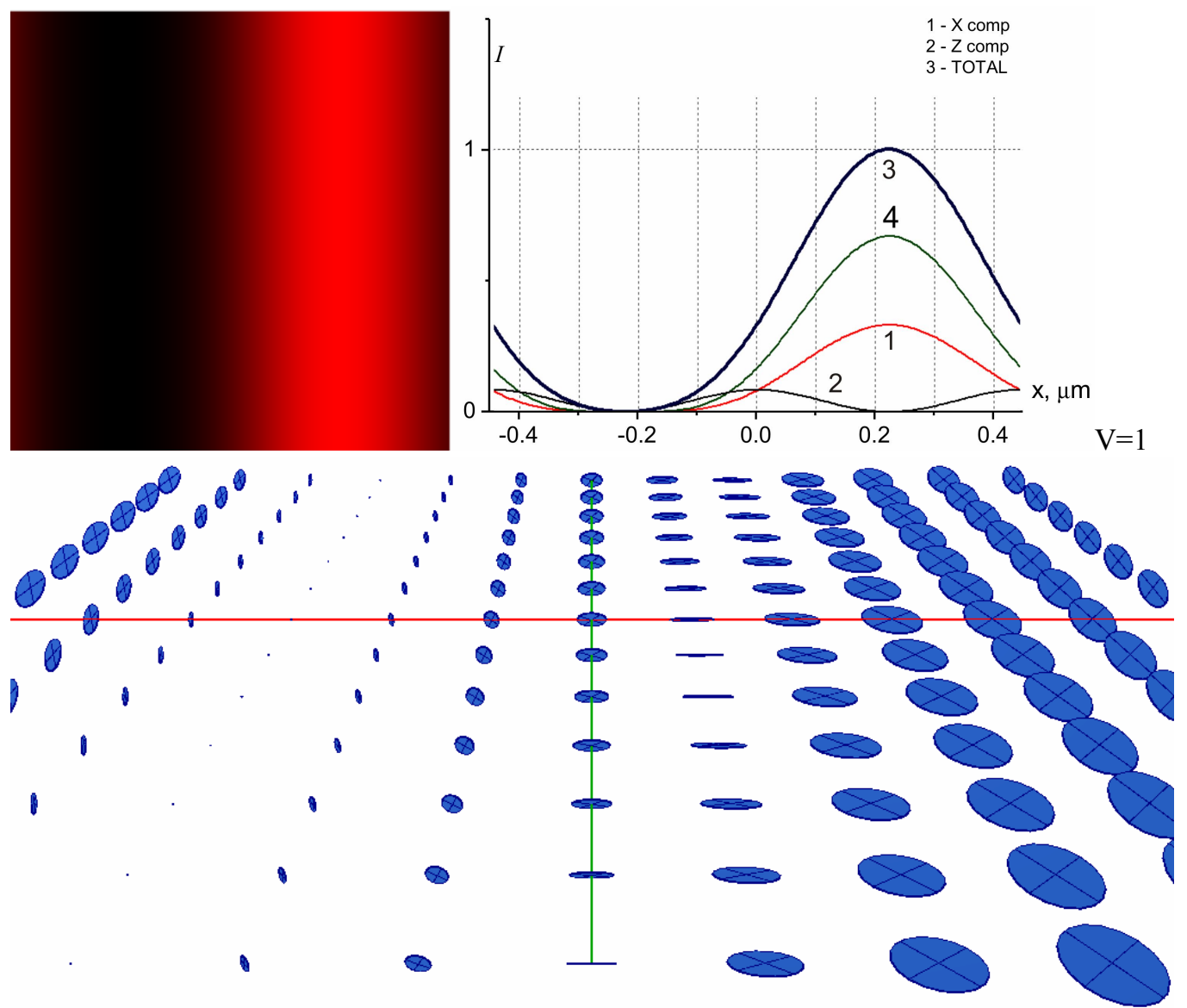

Fig. 5. Intensity distribution and spatial polarisation modulation associated with the interaction of $x-, z$ - and $y$-, $z$-field components in the observation area for the case of superposition of three circularly polarised waves. The visibility modulation depth corresponds to the degree of coherence of the initial waves $\left(M=\left|\eta^{(1,2)}\right|\right)$. The notation is the same as in Fig. 3.

The increase in the azimuth of the reference wave up to $90^{\circ}$ forms a circularly polarised wave, and its superposition with the initial waves provides both a zero phase difference, leading to formation of a linearly polarised state, and zero intensity at certain points of the recording plane.

In this case we achieve the VMD which is equal to unity (Fig. 5). This value corresponds to the degree of coherence of the initial waves. The polarisation modulation 
determined by alignment of the phases of the field components becomes more complex. Then the depth of the polarisation changes, which corresponds to the depth of the intensity modulation, defines exactly the correlation properties of the initial superposing waves.

\section{Conclusions}

We have shown that the technique suggested in the work [6] for determining the coherence properties of orthogonal linearly polarised waves could also be applied for estimating the degree of coherence of circularly polarised coherent waves. Here the characteristic features of the polarisation changes are transformed into the corresponding spatial modulation of the visibility. There is no doubt that the technique proposed in this work and based upon utilization of the circularly polarised waves should be useful for solving many problems of applied polarisation holography and obtaining polarisationsensitive diffraction gratings.

\section{References}

1. Setala T, Tervo J and Friberg A T, 2006. Stokes parameters and polarization contrasts in Young's interference experiment. Opt. Lett. 31: 2208-2210.

2. Setala T, Tervo J and Friberg A.T, 2006. Contrasts of Stokes parameters in Young's interference experiment and electromagnetic degree of coherence. Opt. Lett. 31: 2669-2671.

3. Angelsky O V, Dominikov NN and Maksimyak P P and Tudor T, 1999. Experimental revealing of polarization waves. Appl. Opt. 38: 3112-3117.

4. Angelsky O V, Yermolenko S B, Zenkova C Yu and Angelskaya A O, 2008. On polarization manifestations of correlation (intrinsic coherence) of optical fields. Appl. Opt. 47: 5492-5499.

5. Angelsky O V, Zenkova C Yu, Gorsky M P and Gorodyns'ka N V, 2009. On the feasibility for estimating the degree of coherence of waves at near field. Appl. Opt. 48: 2784-2788.

6. Angelsky O V, Hanson S G, Zenkova C Yu, Gorsky M P and Gorodyns'ka N V, 2009. On polarization metrology (estimation) of the degree of coherence of optical waves. Opt. Express. 17: 15623-15634.

7. Angelsky O V, Besaha R N and Mokhun II, 1997. Appearance of wave front dislocations under interference among beams with simple wave fronts, Optica Applicata. XXVII: 273-278.

8. Gori F 1999. Measuring Stokes parameters by means of a polarization grating, Opt. Lett. 24: 584-586.

9. Cipparrone G, Mazzulla A, Palto S P, Yudin S G and Blinov L M, 2000. Permanent polarization gratings in photosensitive Langmuir-Blodgett films. Appl. Phys. Lett. 77: 2106-2108

10. Rochon P, Drnoyan V and Natansohn A, 1998. Polarization holographic gratings in azopolymers and producing circularly polarized light, Proc. SPIE. 3491: 306-309. 
11. Lagugne-Labarthet F, Rochon P and Natansohn A, 1999 Polarization Analyses of Diffracted Orders from a Birefringence Grating Recorded on Azobenzene Containing Polymer. Appl. Phys. Lett. 75: 1377-1379.

12. Cloutier S G, Peyrot D A, Galstian T V and Lessard R A, 2002. Measurement of permanent vectorial photoinduced anisotropy in azo-dye-doped photoresist using polarization holography. J. Opt. A: Pure Appl. Opt. 4: S228-S234.

13. Holme N C R, Nikolova L, Ramanujam P S and Hvilsted S, 1997. An analysis of the anisotropic and topographic gratings in a side-chain liquid crystalline absobenzene polyester. Appl. Phys. Lett. 70: 1518-1520.

14. Nikolova L, Petrova T, Ivanov M, Todorov T and Nacheva E, 1992. Polarization holographic gratings. Diffraction efficiency of amplitude-phase gratings and their realization in $\mathrm{AgCl}$ emulsions. J. Mod. Opt. 39: 1953-1963.

15. Nikolova L, Todorov T, Ivanov M, Andruzzi F, Hvilsted S and Ramanujam P S, 1996. Polarization holographic gratings in side-chain azobenzene polyesters with linear and circular photoanisotropy, Appl. Opt. 35: 3835-3840.

16. Ciuchi F, Mazzulla A and Cipparrone G, 2002. Permanent polarization gratings in elastomer azo-dye systems: comparison of layered and mixed samples, J. Opt. Soc. Amer. B. 19: 2531-2537.

17. Ono H, Emoto A, Takahashi F, Kawatsuki N and Hasegawa T, 2003. Highly stable polarization gratings in photocrosslinkable polymer liquid crystals, J. Appl. Phys. 94: 1298-1303.

18. Cloutier S G, 2005. Polarization holography: orthogonal plane-polarized beam configuration with circular vectorial photoinduced anisotropy. J. Phys. D: Appl. Phys. 38: $3371-3375$.

19. Emoto A, Ono H, Kawatsuki N, Uchida E and Kuwabara M, 2006. Polarization conversion in polarization holographic gratings formed in photocrosslinkable polymer liquid crystals. AZojomo. 2: $10 \mathrm{p}$.

20. Tervo J, Setala T and Friberg A T, 2003. Degree of coherence for electromagnetic fields. Opt. Express. 11: 1137-1143.

Zenkova C.Yu., Gorsky M.P. and Gorodynska N.V., 2010. About the estimation of degree of coherence for circularly polarized waves. Ukr.J.Phys.Opt. 11: 127-137.

\begin{abstract}
Анотація. У даній роботі нами запропоновано метод поляризаційної модулячії поля для визначення ступеня когерентності ииркулярно поляризованих хвиль. Досліджено прочес трьох-хвильової взаємодії в наближенні проблем прикладної поляризаційної голографії $i$, зокрема, поляризаційно чутливих дифракційних граток. Продемонстровано важливу роль опорної циикклярно поляризованої хвилі у перетворенні просторового розподілу поляризації в глибину модуляиії видності результуючого розподілу, яка може бути метрологічно оцінена і проаналізована.
\end{abstract}

